\title{
Study on Effect Elements of Exopolysaccharide Production of Lactobacillus Kimchi SR8 and DPPH Radical Scavenging Activity
}

\author{
Yulong Zhang, Ping Hu*, Min Fan, Qianwei Liao \\ School of Liquor and Food Engineering, Guizhou University, Guiyang, China \\ *Corresponding author: phu1@gzu.edu.cn
}

\begin{abstract}
The correlation between exopolysaccharide production of Lactobacillus kimchi SR8 under different culture conditions and the DPPH radical scavenging activity of exopolysaccharide was studied. Lactobacillus kimchi SR8 produced $228.24 \pm 2.23 \mathrm{mg} / \mathrm{L}$ exopolysaccharides with sucrose as the carbon source, Beef Extract-Peptone (2:1) as the nitrogen source and an initial $\mathrm{pH}$ of 6.50 , whilst the DPPH radical scavenging activity of exopolysaccharides was only $6.85 \% \pm 0.77 \%$ at a concentration of $0.20 \mathrm{mg} / \mathrm{mL}$. However, the strain produced $206.79 \pm 2.23 \mathrm{mg} / \mathrm{L}$ exopolysaccharides with glucose as the carbon source, Peptone-Tryptone (1:1) as the nitrogen source and an initial $\mathrm{pH}$ of 7.00 , and the DPPH radical scavenging activity of exopolysaccharides increased to $28.34 \% \pm 0.32 \%$ at the same concentration. The results showed no correlation between the exopolysaccharide production of lactic acid bacteria and the DPPH radical scavenging activity of exopolysaccharide. Therefore, the antioxidant activity should be taken into consideration when measures are taken to increase exopolysaccharide production if greater antioxidant activity of exopolysaccharides is preferred.
\end{abstract}

Keywords: characterization, exopolysaccharide, free radical, scavenging activity, relationship

Cite This Article: Yulong Zhang, Ping Hu, Min Fan, and Qianwei Liao, "Study on Effect Elements of Exopolysaccharide Production of Lactobacillus Kimchi SR8 and DPPH Radical Scavenging Activity.” Journal of Food and Nutrition Research, vol. 5, no. 12 (2017): 928-934. doi: 10.12691/jfnr-5-12-8.

\section{Introduction}

Lactic acid bacteria (LAB) exopolysaccharides (EPS) are high-molecular -weight polymers that are produced during growth and reproduction to adapt to the environment $[1,2]$. They are composed of repeating and branched units of sugars or their derivatives, such as glucose, fructose, mannose, lactose, rhamnose and galactose [3,4]. In recent years, LAB EPSs have received increasing attention because LAB strains are generally regarded as safe and of food-grade status [5,6,7]. Thus far, the main LAB strains that have been found to produce EPS are Lactobacillus rhamnosus [8], Lactobacillus plantarum [9], Lactobacillus helveticus [10], Lactobacillus delbrueckii subsp. Bulgaricus [11], Lactococcus lactis subsp. Lactis [12], Bifidobacterium bifidum [13], Pediococcus pentosaceus [14] and Streptococcus thermophiles [15]. The production of EPS is influenced by parameters such as the starter culture, medium, incubation time and extraction methods. A report has shown that a probiotic Lactobacillus plantarum MTCC 9510 produced $0.14 \mathrm{~g} / \mathrm{L}$, but its production was increased up to $1.08 \mathrm{~g} / \mathrm{L}$ when ammonium sulphate was used as an inorganic nitrogen source [1].

Studies have demonstrated that the antioxidant effects of LAB EPS may involve non-enzymatic antioxidant activities, up-regulation of enzymatic antioxidant activities, inhibition of lipid peroxidation, and scavenging of reactive oxygen species $[9,16,17]$. They also scavenge free radicals, including hydroxyl radical, superoxide anion radical and 1,1-diphenyl-2-picrylhydrazyl free radical (DPPH radical). Catalase, superoxide dismutase and glutathione peroxidase activity were increased in the serum and livers of mice treated with EPS and phosphorylated exopolysaccharide (P-EPS) [18]. In addition, EPSs show immunity stimulation, cholesterol reduction, anti-allergy and antitumor activity $[19,20,21,22]$.

Due to the different functions of LAB EPS, such as antioxidant activity, anticancer activity and dairy stabilization, measures have been taken to increase EPS production. After observing the anticancer activity of LAB EPSs, Deepak et al. [23] used response surface methods to increase cancer EPS production from Lactobacillus acidophilus, and the level of EPS increased to $597 \mathrm{mg} / \mathrm{L}$. The optimization models and methods were developed only to enhance EPS production. Desai et al. [24] applied artificial intelligence-based techniques in the optimization of fermentation media to enhance the EPS production from Lactobacillus plantarum and increased production to $7.14 \mathrm{~g} / \mathrm{L}$. Further studies include the optimization of EPS production and the functions of EPS from the optimized culture conditions. Hsieh et al. [25] optimized Lactobacillus acidophilus cultivation using taro waste and evaluated the anti-tumour and immunomodulatory activities of heatkilled cells, cytoplasmic fraction and EPSs extracted from culture in the optimized conditions. However, most studies have focused only on EPS production or functions and 
neglected EPS activities in the same conditions and few reports have combined EPS production with antioxidant activity.

In our previous studies, a LAB strain of Lactobacillus kimchi SR8 was screened from 36 LAB strains isolated from fermented meat products. This strain has high EPS production ability and the EPS also has antioxidant activity. The aim of this study was to use Lactobacillus kimchi SR8 as the research strain to explore the relationship between EPS production extracted from different LAB culture conditions and the DPPH radical scavenging activities of EPS. The DPPH radical scavenging activity was used as an indicator of antioxidant activity of EPS. Much attention has been paid if greater antioxidant activity of EPS is preferred when measures are taken to increase EPS production of LAB.

\section{Materials and Methods}

\subsection{LAB Strain}

Lactobacillus kimchi SR8 was isolated and identified from traditionally fermented sour meat from China and was screened from 36 strains of LAB and identified as a strain with high production of EPS [26]. The strain was maintained at $4^{\circ} \mathrm{C}$ and renewed for 4 weeks for short-term preservation. In the study, it was inoculated in MRS broth (Beijing Land Bridge Co., Ltd) two or three times to measure the production and DPPH radical scavenging activity of its EPS.

\subsection{Growth Characteristics}

Lactobacillus kimchi SR8 was grown in fresh MRS broth with an inoculum size of $3 \%(\mathrm{v} / \mathrm{v})$. All samples were cultivated at $37^{\circ} \mathrm{C}$ from $0 \mathrm{~h}$ to $24 \mathrm{~h}$. The absorbance at $600 \mathrm{~nm}\left(\mathrm{~A}_{600}\right)$, soluble solid proportion (SSP) and $\mathrm{pH}$ values were measured respectively using T6 New Century UV-Vis spectro-photometer (Beijing Persee General Instrument Co., Ltd), WZS-20 Portable Refractometer (Shanghai physical optics instrument Co., Ltd) and Starter 3100 pH meter (Ohaus Instrument (Shanghai) Co., Ltd).

\subsection{Production Properties of Exopolysaccharide}

Lactobacillus kimchi SR8 was grown in fresh MRS broth with an inoculum size of $3 \%(\mathrm{v} / \mathrm{v})$. A third of all samples were cultivated at $37^{\circ} \mathrm{C}$ from $26 \mathrm{~h}$ to $34 \mathrm{~h}$. One third of samples were cultivated respectively at 33, 35, 37, 39 and $41^{\circ} \mathrm{C}$ for $24 \mathrm{~h}$. The $\mathrm{pH}$ values of the last one third of samples were respectively regulated to 5.50, 6.00, 6.50, 7.00 and 7.50 and those were cultivated at $37^{\circ} \mathrm{C}$ for $24 \mathrm{~h}$. The EPS-producing capacity on conditions of culture time, temperature and $\mathrm{pH}$ were measured.

\subsection{Exopolysaccharide Determination}

Fermented culture with Lactobacillus kimchi SR8 was centrifuged at $10000 \mathrm{rpm}$ at $4^{\circ} \mathrm{C}$ for $20 \mathrm{~min}$. The protein was then precipitated from the supernatant overnight by the addition of $80 \%$ trichloroacetic acid solution at a final concentration of $10 \%$ and centrifuged at $10000 \mathrm{rpm}$ at $4^{\circ} \mathrm{C}$ for 20 min for removal. Furthermore, small molecular carbohydrates were dialysed out of the dialysis bag MD34 (Molecular cut off: 8 000-14 000, Solarbio, Beijing) by overnight dialysis. The dialyzed solution was added to triple volumes of absolute ethanol (Tianjin Fuyu, Tianjin) to precipitate EPS and then centrifuged at $10000 \mathrm{rpm}$ at $4^{\circ} \mathrm{C}$ for $20 \mathrm{~min}$. The precipitated EPS was dissolved for measurement of the contents. The absorbance of the EPS sample solution was measured at $490 \mathrm{~nm}$ with a phenyl hydroxide (Sinopharm chemical reagent Co. Ltd, Shanghai)-sulfuric acid (Chuandong Chemical, Chongqing) procedure [20] and calibrated using glucose as a standard.

\subsection{Free Radical Scavenging Activity}

The DPPH radical scavenging activities of EPSs were measured with Choi's method [21]. The concentration of EPSs was respectively regulated to $0.08,0.16,0.24,0.32$ and $0.40 \mathrm{mg} / \mathrm{mL}$ and then all samples were used to determine the radical scavenging activity. The DPPH radical scavenging activity of EPS was calculated with the following equation:

$$
\begin{aligned}
& \text { Scavenging activity (\%) } \\
& =\left(1-\frac{A_{517} \text { of sample }}{A_{517} \text { of control }}\right) \times 100 \text {. }
\end{aligned}
$$

\subsection{Relationship between EPS Production and Radical Scavenging Activity}

\subsubsection{Effect of Carbon Source on EPS Production and the DPPH Radical Scavenging Activity}

Lactobacillus kimchi SR8 was inoculated in fresh modified MRS culture including lactose, glucose, maltose or sucrose as carbon source with an inoculum size of $1 \%$. All samples were inoculated at $37^{\circ} \mathrm{C}$. After $24 \mathrm{~h}$ of incubation at $37^{\circ} \mathrm{C}$, the culture was centrifuged at 10000 $\mathrm{rpm}$ at $4^{\circ} \mathrm{C}$ for $20 \mathrm{~min}$ to remove the LAB cells. EPSs were then obtained by addition of $80 \%$ trichloroacetic acid solution for precipitating proteins and by dialysis to remove small molecular carbohydrates. The precipitated EPS was used to measure the DPPH radical scavenging activity at the EPS concentration of $0.16 \mathrm{mg} / \mathrm{mL}$.

\subsubsection{Effect of Nitrogen Source on EPS Production and the DPPH Radical Scavenging Activity}

Lactobacillus kimchi SR8 was grown in fresh modified MRS culture including beef extract-peptone (1:2, $1: 1$ and $2: 1)$, beef extract-tryptone $(1: 1)$ or peptone-tryptone $(1: 1)$ as nitrogen source with an inoculum size of $1 \%$. After 24 $\mathrm{h}$ of incubation at $37^{\circ} \mathrm{C}$, the EPS was precipitated by centrifugation to remove cells, precipitation of proteins by the addition of $80 \%$ trichloroacetic acid and dialysis for the removal of small molecular carbohydrates. The DPPH radical scavenging activity of precipitated EPS was measured at $517 \mathrm{~nm}$ at the EPS concentration of $0.16 \mathrm{mg} / \mathrm{mL}$.

\subsubsection{Effect of pH on EPS Production and the DPPH Radical Scavenging Activity}

Lactobacillus kimchi SR8 was inoculated in fresh culture adjusted to $\mathrm{pH}$ values of 5.50, 6.00, 6.50, 7.00 and 
7.50 with an inoculum size of $1 \%$. After $24 \mathrm{~h}$ of incubation at $37^{\circ} \mathrm{C}$, the EPS was extracted from the cultures with different $\mathrm{pH}$ values to examine DPPH radical scavenging activity at the EPS concentration of $0.16 \mathrm{mg} / \mathrm{mL}$.

\subsection{Statistical Analysis}

All of determinations were carried out in triplicate, and all mean values were used for statistical analysis. Analysis of variance was performed with SPSS software (version 16.0, IBM, USA).

\section{Results}

\subsection{Growth Characteristics of Lactobacillus kimchi SR8}

Adequate nutrients improve growth and increase the production of active substances. The fermentation characteristics of Lactobacillus kimchi SR8 were measured with MRS broth as a substrate (Figure 1). With extension of the culture time, the absorbance at $600 \mathrm{~nm}$ showed an ascendant trend and reached its absorbance peak value $(2.359 \pm 0.007)$ when the culture time reached the tenth hour. It illustrated an adaptive phase in the range of 0 to $4 \mathrm{~h}$ when the activated culture was homogenized with fresh culture, a logarithmic phase in the range of 4 to $8 \mathrm{~h}$ because of adequate nutritious substrates and a stationary phase in the range of 8 to $24 \mathrm{~h}$ due to the consumption of substrates (Figure 1a). With continuing fermentation, LAB strains consumed substrates for growth and reproduction so that the soluble composition content decreased. The soluble solid proportion of zymotic fluid declined until the culture time reached the eighth hour and then reached a plateau as the biomass changed. The initial $\mathrm{pH}$ value of the zymotic fluid was $6.47 \pm 0.05$. Along with the reproduction and fermentation from the isolate, the $\mathrm{pH}$ gradually decreased and then reached a plateau when the culture time reached the eighth hour. At that moment, the reproduction and growth of the isolate also remained steady. Therefore, acid production mainly appeared during the reproduction and growing phases. The $\mathrm{pH}$ of the zymotic fluid changed from an initial value of $6.47 \pm 0.05$ to a steady-state value of $4.86 \pm 0.01$ (Figure $1 \mathrm{~b}$ ).

\subsection{Effect of Incubation Time, Temperature and $\mathrm{pH}$ on Production}

The glucose standard curve for EPS production $(\mathrm{y}=0.0112 \mathrm{x}+0.0271 ; \mathrm{R} 2=0.99997)$ illustrates fitness for the calculation of EPS production (Figure 2).

As illustrated in Figure 3a, the EPS production from Lactobacillus kimchi SR8 increased slowly from the 26th to the 34th hour and reached a peak value of $210.37 \pm 3.57$ $\mathrm{mg} / \mathrm{L}$ at the 32nd hour. Afterward, the production of EPS showed a declining trend. The growing environment has a significant effect on secretion of EPS. A harsh environment can enhance the secretion of some functional compounds of microorganisms. The EPS production changed as fermentation temperature rose (Figure $3 b$ ).
When fermentation temperature increased, the production of EPS produced by LAB gradually increased. The LAB produced EPS that was used for their survivals to resist the high-temperature environment. The EPS production reached $236.28 \pm 1.79 \mathrm{mg} / \mathrm{L}$ when fermentation temperature was up to $41^{\circ} \mathrm{C}$. The $\mathrm{pH}$ also showed effects on the secretion of EPS of the isolate Lactobacillus kimchi SR8 (Figure 3c). The alkaline conditions inhibited the secretion of EPS of Lactobacillus kimchi SR8. It was drawn that the secretion of EPS raised just on suitable conditions.

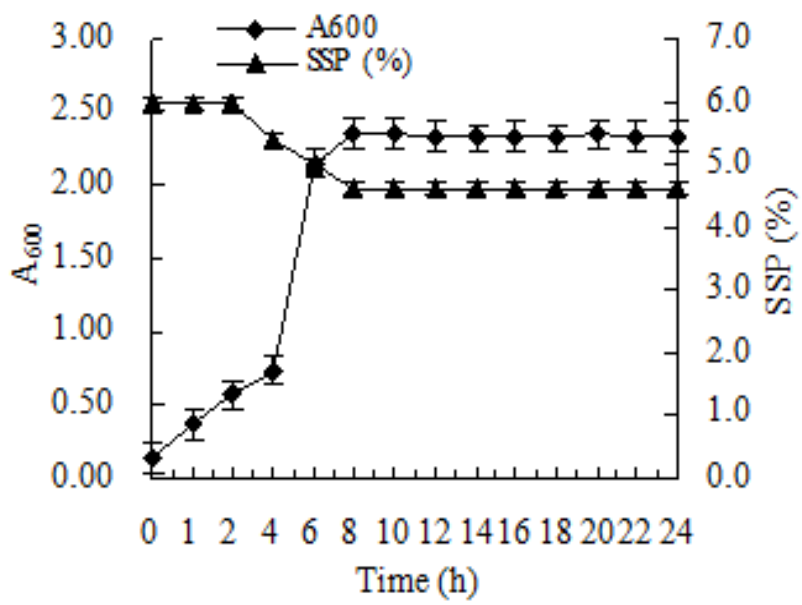

a. Growth characteristics of LAB strain

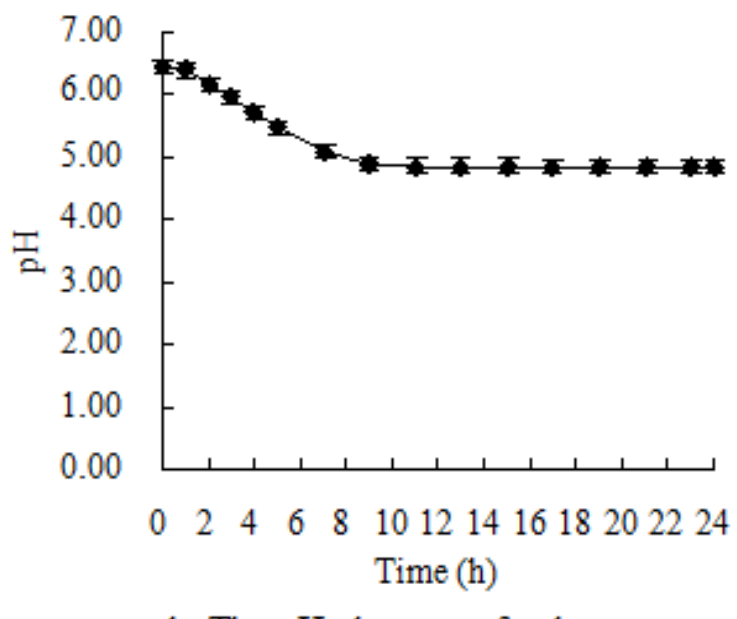

b. The $\mathrm{pH}$ changes of culture

Figure 1. Growth and acid-producing properties of Lactobacillus kimchi SR8

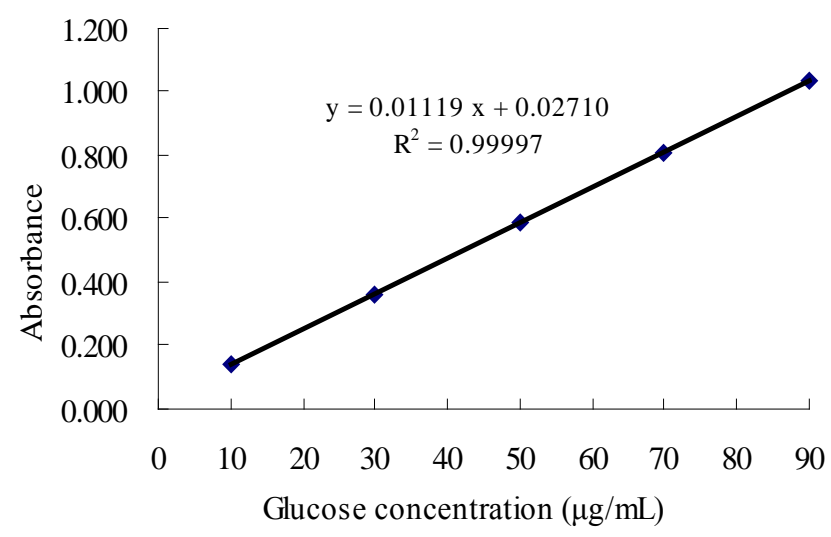

Figure 2. The glucose standard curve 


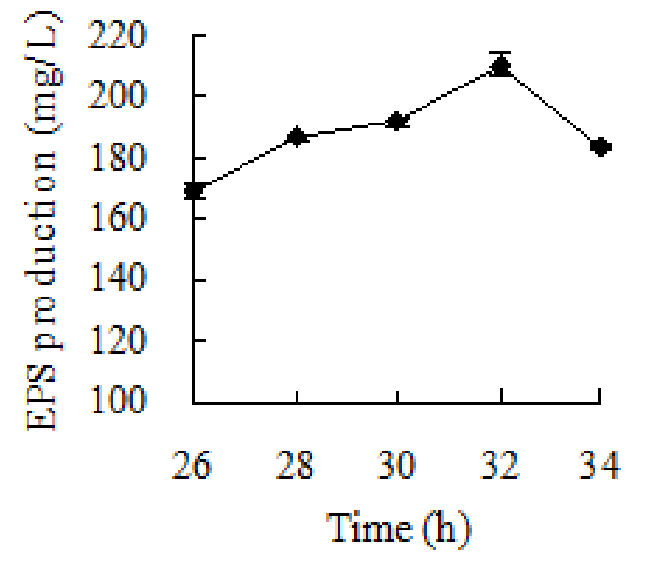

a. Effect of time on EPS production

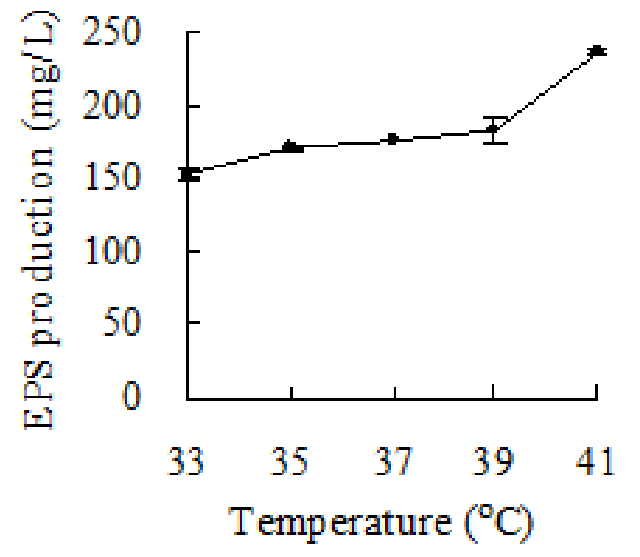

b. Effect of temperature on EPS production

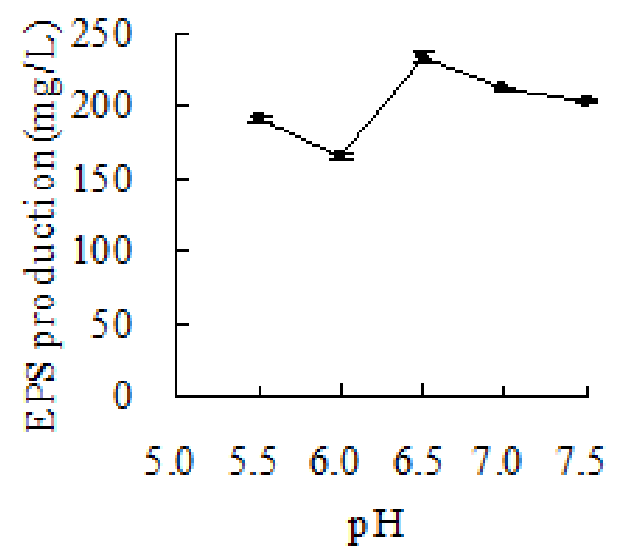

\section{c. Effect of $\mathrm{pH}$ on EPS production}

Figure 3. Effect of incubation time and temperature on EPS production

\subsection{Free Radical Scavenging Activity}

DPPH radical scavenging activity was chosen as a free radical scavenging activity index for antioxidant activity of EPS. The DPPH radical scavenging activity of EPS at different concentrations was shown in Figure 4. With EPS concentration rising, the DPPH radical scavenging activity of EPS rose steadily. The scavenging activity suddenly rose rapidly when EPS concentration exceeded $0.32 \mathrm{mg} / \mathrm{mL}$.

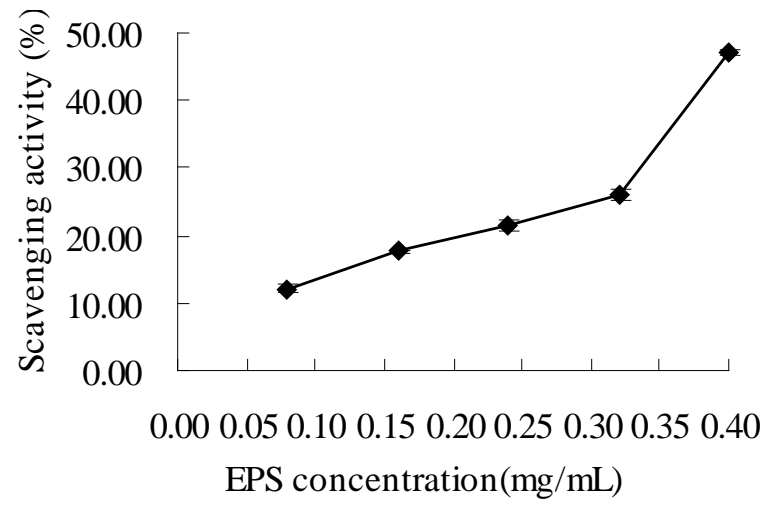

Figure 4. Effect of EPS concentration on DPPH radical scavenging activity

\subsection{Relationship between Production and Free Radical Scavenging Activity}

\subsubsection{Effect of Carbon Source on Production and DPPH Radical Scavenging Activity}

To select the optimal carbon source for EPS production and DPPH radical scavenging activity, the glucose in the MRS broth was replaced with lactose, maltose or sucrose (a final concentration, $20.00 \mathrm{~g} / \mathrm{L}$ ). The different kinds of sugar showed different effects on EPS production and DPPH radical scavenging activity. Sucrose showed the most effective carbon source for the production of LAB EPS (263.84 $\pm 2.98 \mathrm{mg} / \mathrm{L})$. However, although EPS production was the greatest, the DPPH radical scavenging activity was the slowest than those of other carbon sources. According to Figure 5, EPS from fermented supernatants with glucose as the carbon source showed a higher DPPH radical scavenging activity (17.95 $\pm 0.36 \%)$. The EPS from the three kinds of reducing monosaccharides showed a good DPPH radical scavenging activity, while EPS from a non-reducing disaccharide (sucrose) illustrated a low DPPH radical scavenging activity $(7.84 \pm 2.35 \%)$.

\subsubsection{Effect of nitrogen source on production and DPPH radical scavenging activity}

The nitrogen source, a vital growth factor to $\mathrm{LAB}$, showed an insignificant influence on their physiological-biochemical characteristics. This study showed the production and DPPH radical scavenging activity of EPS with different nitrogen sources and their ratios (Figure 6). The production of EPS was highly enhanced $(284.99 \pm 3.18 \mathrm{mg} / \mathrm{L})$ when the nitrogen source was BP2:1, followed by those with BP1:2, BT1:1 and PT1:1 as nitrogen sources. Compared to former production of EPS, the production of the former EPS production was more than 19\%. However, EPS production from different nitrogen sources was shown to be irrelevant to its DPPH radical scavenging activity at the same final EPS concentration. EPS from BP2:1 used as a nitrogen source illustrated higher production and lower DPPH radical scavenging activity than those from other nitrogen source.

In addition, EPS from PT1:1 culture showed a great $\mathrm{DPPH}$ radical scavenging activity (31.03 $\pm 0.90 \%)$, followed by those of EPSs from those with BP1:2, BP1:1 and BT1:1 as nitrogen sources, with an EPS production of $245.67 \pm 2.91 \mathrm{mg} / \mathrm{L}$. The nitrogen sources and their ratios 
had a significant effect on the EPS's DPPH radical scavenging activity.

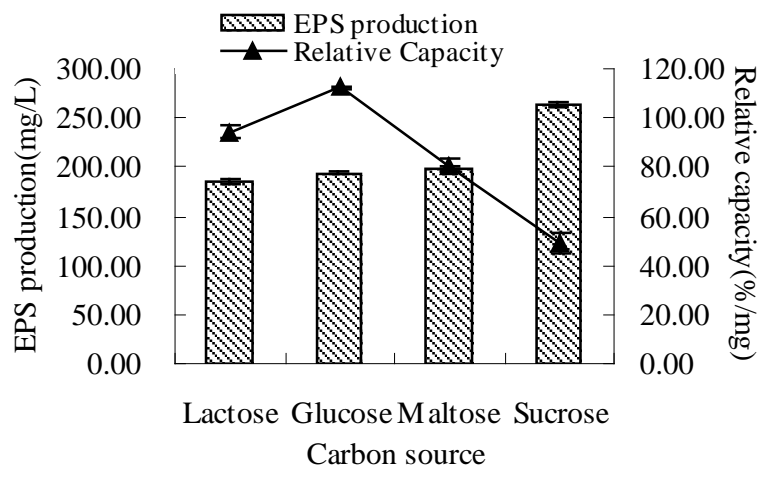

Figure 5. Influence of carbon source on the production of EPS and DPPH radical scavenging activity

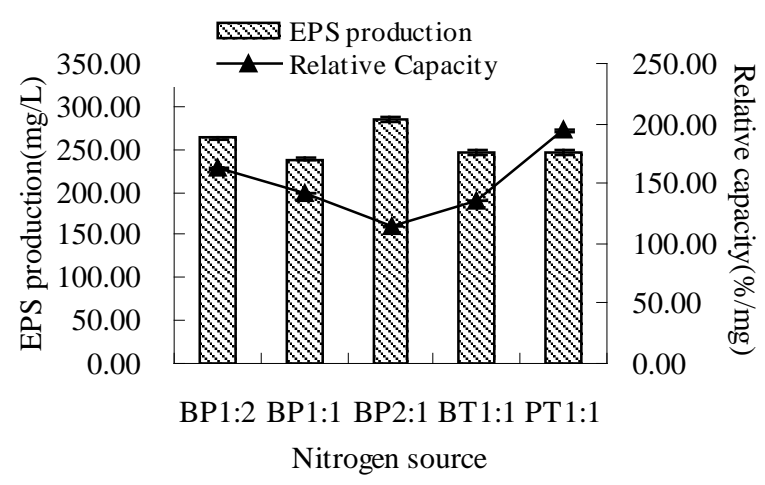

Figure 6. Influence of nitrogen source on the production of EPS and DPPH radical scavenging activity

Note: BP1:2, BP1:1, BP2:1, BT1:1 and PT1:1 are respectively represented as Beef Extract-Peptone (1:2), Beef Extract-Peptone (1:1), Beef Extract-Peptone (2:1), Beef Extract-Tryptone (1:1) and Peptone-Tryptone (1:1).

\subsubsection{Effect of pH on Production and DPPH Radical Scavenging Activity}

About effect of $\mathrm{pH}$ on microbial EPS, $\mathrm{pH}$ may stimulate the activities of enzymes and intermediates. LAB EPS production showed differences due to the initial $\mathrm{pH}$ values of the cultures (Figure 7). The EPS production showed a trend from rising to declining. EPS production reached a peak at a pH of $6.5(234.05 \pm 3.57 \mathrm{mg} / \mathrm{L})$. With the $\mathrm{pH}$ rising continuously, the EPS production showed downtrend.

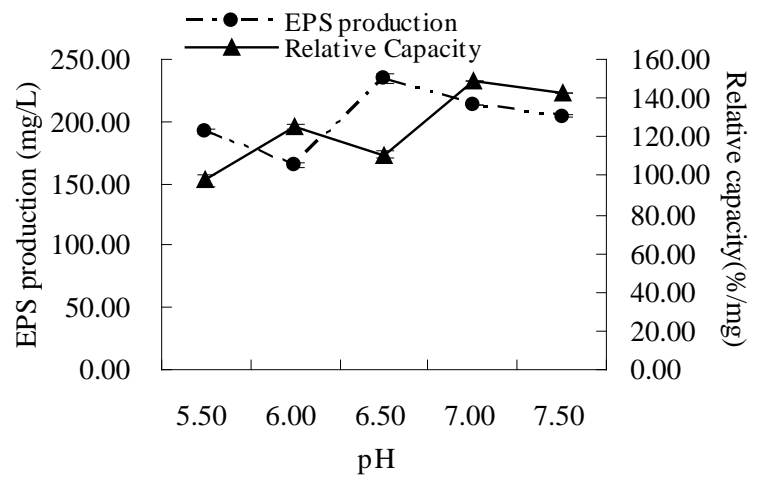

Figure 7. Influence of $\mathrm{pH}$ value on the production of EPS and DPPH radical scavenging activity
In addition, the DPPH radical scavenging activity of EPS showed differences in the different initial $\mathrm{pH}$ values of the cultures. As the $\mathrm{pH}$ increased, the scavenging activity showed an increasing trend (Fig.7). The EPS extracted from the medium with an initial $\mathrm{pH}$ of 7.00 showed the highest DPPH radical scavenging activity $(23.85 \pm 0.00 \%)$. When the $\mathrm{pH}$ value rose continuously, the scavenging activity weakened. It illustrated that the initial $\mathrm{pH}$ value had an effect on the $\mathrm{DPPH}$ radical scavenging activity of EPS.

\subsection{Production and Radical Scavenging Activity of Exopolysaccharides on Combined Conditions}

With reference to the effects of the carbon source, nitrogen source and initial $\mathrm{pH}$ value on $\mathrm{DPPH}$ radical scavenging activities, the production and DPPH radical scavenging activity of EPS from the combination of the following conditions were measured (Table 1). Lactobacillus kimchi SR8 produced $228.24 \pm 2.23 \mathrm{mg} / \mathrm{L}$ EPS with sucrose as the carbon source, BP2:1 as the nitrogen source and an initial $\mathrm{pH}$ 6.50, and the EPS's DPPH radical scavenging activity was just $6.85 \% \pm 0.77 \%$ with a 0.20 $\mathrm{mg} / \mathrm{mL}$ EPS concentration. However, Lactobacillus kimchi SR8 produced $206.79 \pm 2.23 \mathrm{mg} / \mathrm{L}$ EPS with glucose as the carbon source, PT1:1 as the nitrogen source and an initial $\mathrm{pH}$ of 7.00 , and the $\mathrm{DPPH}$ radical scavenging activity reached $28.34 \% \pm 0.32 \%$ in the same conditions. These combined test results verify the previous finding that the effect elements of the carbon source, the nitrogen source and the initial $\mathrm{pH}$ value have different influences on the EPS production of Lactobacillus kimchi SR8 and the DPPH radical scavenging activity.

Table 1. Production and DPPH radical scavenging activity of EPS from different culture conditions

\begin{tabular}{ccccc}
\hline & & & \multicolumn{2}{c}{ Results } \\
\cline { 4 - 5 } Carbon & Nitrogen & pH & $\begin{array}{c}\text { EPS Production } \\
(\mathrm{mg} / \mathrm{L})\end{array}$ & $\begin{array}{c}\text { DPPH radical } \\
\text { scavenging activity (\%) }\end{array}$ \\
\hline Sucrose & BP2:1 & 6.50 & $228.24 \pm 2.23$ & $6.85 \pm 0.77$ \\
Glucose & PT1:1 & 7.00 & $206.79 \pm 2.23$ & $28.34 \pm 0.32$ \\
\hline
\end{tabular}

Note: BP2:1 and PT1:1 are represented as Beef Extract-Peptone (2:1) and Peptone-Tryptone (1:1), respectively.

\section{Discussion}

Many LAB isolates, such as Lactobacillus sp. Ca6, Lactobacillus kefiranofaciens, Lactobacillus plantarum and Lactobacillus rhamnosus, can produce EPS [4,5,6,8,9]. The EPS produced by lactobacilli was an important functional compound for their survivals in hypertonic environment [29]. Reported lactobacilli with producing EPS were approximately 30 species [6]. However, EPS production of lactic acid bacteria was influenced by many factors, such as medium components, culture conditions, initial $\mathrm{pH}$ value and so on except for species of LAB. Wang's study showed a great influence of fermentation temperature, initial $\mathrm{pH}$ and nitrogen source on EPS production [30]. In our study, EPS production was also influenced by fermentation temperature and time, initial $\mathrm{pH}$ and medium. Imran et al. reported that the EPS 
production of Lactobacillus plantarum reached a maximum in stationary phase of growth (32 h), and then decreased in declining phase [31], which was consistent with our results. This decrease in EPS production after prolonged culture was reported earlier [32]. For the effect of $\mathrm{pH}$ value, $\mathrm{pH}$ may stimulate the activities of enzymes and intermediates. It has been reported that lipid intermediate has an important effect on the biosynthesis of microbial polysaccharides. With the $\mathrm{pH}$ changing, EPS production changed and reached the maximum $234.05 \pm$ $3.57 \mathrm{mg} / \mathrm{L}$ at $\mathrm{pH}$ 6.50, which was same to initial $\mathrm{pH}$ (pH 6.5) for the maximum production of EPS from Lactococcus lactis subsp. Lactis reported in Tao's study [33], which was consistent with the results of Lactobacillus kimchi SR8 in this study. Ibarburu et al. [34] studied production and partial characterization of EPS from Lactobacillus suebicus isolates, and found that EPS production was influenced by fermentation time, initial $\mathrm{pH}$ and LAB strains. As potential functional composition, antioxidant activity of EPS from LAB received much attention. The scavenging activity of DPPH radical was chosen to evaluate antioxidant activity of EPS in this study due to that DPPH radical, a synthetic free radical, has a proton free radical with a characteristic absorption, which significantly decreased when it was exposed to proton radical scavengers when it was compared to other free radicals [35]. It was found that the scavenging activity of DPPH radical of exopolysaccharides from Lactobacillus kimchi SR8 was up to $47.20 \%$ at 0.40 $\mathrm{mg} / \mathrm{mL}$. Zhang et al. [9] reported antioxidant activity of EPS from Lactobacillus plantarum C88, and its scavenging activity of DPPH radical was influenced by EPS concentration and the activity was about $43 \%$ at 1 $\mathrm{mg} / \mathrm{mL}$, which was lower than EPS's activity in our study. Based on antioxidant activity of EPS, the production of EPS has been optimized with an orthogonal experimental design, response surface method and artificial intelligence-based techniques [23,24,30], but optimization of antioxidant activity of EPS is still not seen. No research shows relationship between optimization of EPS production and its antioxidant activity. In our study, no significant relationship between production and antioxidant activity was found, and EPS production on combined conditions just for production was higher than that on combined conditions only for antioxidant activity of EPS while antioxidant activity of EPS from the former fermentation conditions was far lower than that from the later conditions. Antioxidant activity of EPS should be taken into consideration at the same time when optimization of EPS production is carried out. For that, the aim of high production and high antioxidant activity of EPS will be reached to ensure further study and development of antioxidant EPS products.

\section{Conclusions}

The parameters, including the fermentation time, temperature, initial $\mathrm{pH}$, carbon source and nitrogen source, played important roles in the EPS production of LAB. Lactobacillus kimchi SR8-producing acid mainly appeared during the reproduction and growing phases, and the EPS production reached a peak value $(210.37 \pm 3.57 \mathrm{mg} / \mathrm{L})$ at the 32nd hour and increased further to $236.28 \pm 1.79 \mathrm{mg} / \mathrm{L}$ when cultured at $41^{\circ} \mathrm{C}$. Lactobacillus kimchi SR8 was then studied to improve EPS production under different conditions, including carbon source, nitrogen source and the initial $\mathrm{pH}$ value of the medium. The antioxidant activity of EPS was taken into consideration when optimization was carried out to enhance the quantity of EPS. The results from single-factor experiments and combined test showed no positive correlation between the EPS production of LAB and its DPPH radical scavenging activity. So far, few reports have combined EPS production with antioxidant activity.

\section{Acknowledgements}

This work was supported by National Science Foundation of China program (31260379).

\section{Abbreviations}

$\begin{array}{ll}\text { LAB } & \text { lactic acid bacteria } \\ \text { EPS } & \text { exopolysaccharide } \\ \text { DPPH } & \text { 1,1-diphenyl-2-picrylhydrazyl } \\ \text { SSP } & \text { soluble solid proportion }\end{array}$

\section{References}

[1] Ismail, B., Nampoothiri, K.M., Production, purification and structural characterization of an exopolysaccharide produced by a probiotic Lactobacillus plantarum MTCC 9510, Archives of Microbiology, 192(12). 1049-1057. December 2010.

[2] Surayot, U., Wang, J.G.., Seesuriyachan, P., Kuntiya, A., Tabarsa, M., Lee, Y., Kim, J.K., Park, W.J., You, S., Exopolysaccharides from lactic acid bacteria: Structural analysis, molecular weight effect on Immunomodulation, $\mathrm{k}$ International Journal of Biological Macromolecules, 68. 233-240. July 2014.

[3] Kodali, V.P., Perali, R.S., Sen, R., Purification and partial elucidation of the structure of an antioxidant carbohydrate biopolymer from the probiotic bacterium Bacillus coagulans RK02, Journal of Natural Products, 74(8). 1692-1697. August 2011.

[4] Trabelsi, I., Ben Slima, S., Chaabane, H., Riadh, B.S., Purification and characterization of a novel exopolysaccharides produced by Lactobacillus sp. Ca6, International Journal of Biological Macromolecules, 74. 541-546. March 2015.

[5] Ahmed, Z., Wang, Y.P., Anjum, N., Ahmad, A., Khan, S.T., Characterization of exopolysaccharide produced by Lactobacillus kefiranofaciens ZW3 isolated from Tibet kefir - part II, Food Hydrocolloids, 30(1). 343-350. January 2013.

[6] Badel, S., Bernardi, T., Michaud, P., New perspectives for Lactobacilli exopolysaccharides, Biotechnology Advances, 29(1). 54-66. January-February 2011.

[7] Nadkarni, M.A., Chen, Z.L., Wilkins, M.R., Hunter N., Comparative Genome Analysis of Lactobacillus rhamnosus Clinical Isolates from Initial Stages of Dental Pulp Infection: Identification of a New Exopolysaccharide Cluster, PLOS ONE, 9(3). 1-14. March 2014

[8] Shao, L., Wu, Z.J., Zhang, H., Chen, W., Ai, L.Z., Guo, B.H., Partial characterization and immunostimulatory activity of exopolysaccharides from Lactobacillus rhamnosus KF5, Carbohydrate Polymers, 107. 51-56. July 2014.

[9] Zhang, L., Liu, C.H., Li, D., Zhao, Y.J., Zhang, X., Zeng, X.P., Yang, Z.N., Li, S.Y., Antioxidant activity of an exopolysaccharide isolated from Lactobacillus plantarum C88, International Journal of Biological Macromolecules, 54. 270-275. March 2013.

[10] Li, W., Ji, J., Rui, X., Yu, J.J., Tang, W.Z., Chen, X.H., Jiang, M., Dong, M.S., Production of exopolysaccharides by Lactobacillus helveticus MB2-1 and its functional characteristics in vitro, LWT-Food Science and Technology, 59(2). 732-739. December 2014. 
[11] Grobben, G.J., Chin-Joe, I., Kitzen, V.A., Boels, I.C., Boer, F., Sikkema, J., De Bont, J.A.M., Enhancement of Exopolysaccharide Production by Lactobacillus delbrueckii subsp. bulgaricus NCFB 2772 with a Simplified Defined Medium, Applied and Environmental Microbiology, 64(4). 1333-1337. April 1998.

[12] Pan, D.D., Mei, X.M., Antioxidant activity of an exopolysaccharide purified from Lactococcus lactis subsp. lactis 12, Carbohydrate Polymers, 80(3). 908-914. May 2010.

[13] Li, S.J., Huang, R.H., Shah, N.P., Tao, X.Y., Xiong, Y.H., Wei, H., Antioxidant and antibacterial activities of exopolysaccharides from Bifidobacterium bifidum WBIN03 and Lactobacillus plantarum R315, Journal of Dairy Science, 97(12). 7334-7343. December 2014.

[14] Shin, J.S., Jung, J.Y., Lee, S.G., Shin, K.S., Rhee, Y.K., Lee, M.K., Hong, H.D., Lee, K.T., Exopolysaccharide fraction from pediococcus pentosaceus KFT18 induces immunostimulatory activity in macrophages and immunosuppressed mice, Journal of Applied Microbiology, 120(5). 1390-1402. May 2016.

[15] Zhang, T.H., Zhang, C.H., Li, S.Y., Zhang, Y.C., Yang, Z.N., Growth and exopolysaccharide production by streptococcus thermophilus ST1 in skim milk, Brazilian Journal of Microbiology, 42(4). 1470-1478. October-December 2011.

[16] Liu C.F., Tseng K.C., Chiang S.S., Lee B.H., Hsu W.H., Pan T.M., Immunomodulatory and antioxidant potential of Lactobacillus exopolysaccharides, Journal of the Science of Food and Agriculture, 91(12), 2284-2291. May 2011.

[17] Li J.Y., Jin M.M., Meng J., Gao S,M., Lu R.R., Exopolysaccharide from Lactobacillus planterum LP6: Antioxidation and the effect on oxidative stress, Carbohydrate Polymers, 98, 1147-1152. October 2013.

[18] Guo, Y.X., Pan, D.D., Sun, Y.Y., Xin, L.Y., Li, H., Zeng, X.Q., Antioxidant activity of phosphorylated exopolysaccharide produced by Lactococcus lactis subsp. Lactis, Carbohydrate Polymers, 97(2). 849-854. September 2013.

[19] Ruas-Madiedo P., Hugenholtz J., Zoon P., An overview of the functionality of exopolysaccharides produced by lactic acid bacteria, International Dairy Journal, 12(2-3), 163-171. 2002.

[20] Gorska S., Jachymek W., Rybka J., Strus M., Heczko P.B., Gamian A., Structural and immunochemical studies of neutral exopolysaccharide produced by Lactobacillus johnsonii 142, Carbohydrate Research, 345(1), 108-114. January 2010.

[21] Pan D.D., Mei X.M., Antioxidant activity of an exopolysaccharide purified from Lactococcus lactis subsp. lactis 12, Carbohydrate Polymers, 80(3), 908-914. May 2010.

[22] Gorska S., Schwarzer M., Srutkova D., Hermanova P., Brzozowska E., Kozakova H., Gamian A., Polysaccharides L900/2 and L900/3 isolated from Lactobacillus rhamnosus LOCK 0900 modulate allergic sensitization to ovalbumin in a mouse model, Microbial Biotechnology, 10(3), 586-593. February 2017.

[23] Deepak, V., Pandian, S.R.K., Sivasubramaniam, S.D., Nellaiah, H., Sundar, K., Optimization of anticancer exopolysaccharide production from probiotic Lactobacillus acidophilus by response surface methodology, Preparative Biochemistry \& Biotechnology, 46(3), 288-297. 2016.
[24] Desai, K.M., Akolkar, S.K., Badhe, Y.P., Tambe, S.S., Lele, S.S., Optimization of fermentation media for exopolysaccharide production from Lactobacillus plantarum using artificial intelligence-based techniques, Process Biochemistry, 41(8). 18421848. August 2006.

[25] Hsieh, S.C., Liu, J.M., Pua, X.H., Ting ,Y.W., Hsu, R.J., Cheng, K.C., Optimization of Lactobacillus acidophilus cultivation using taro waste and evaluation of its biological activity, Applied Microbiology and Biotechnology, 100(6). 2629-2639. March 2016.

[26] Zhang Y.L., Hu P., Wang J.L., Liao Q.W., Isolation of exopolysaccharides-producing lactic acid bacteria and its antioxidant properties, China Brewing, 34(10), 37-42. October 2015.

[27] Zhang, C.H., Yun, Y.H., Fan, W., Liang, Y.Z., Yu, Y., Tang, W.X., Rapid analysis of polysaccharides contents in Glycyrrhiza by near infrared spectroscopy and chemometrics, International Journal of Biological Macromolecules, 79. 983-987. August 2015.

[28] Choi, C.W., Kim, S.C., Hwang, S.S., Choi, B.K., Ahn, H.J., Lee, M.Y., Park, S.H., Kim, S.K., Antioxidant activity and free radical scavenging capacity between Korean medicinal plants and flavonoids by assay-guided comparison, Plant Science, 163(6). 1161-1168. December 2002.

[29] Jindal, N., Singh, D.P., Khattar, J.I.S., Kinetics and physicochemical characterization of exopolysaccharides produced by the cyanobacterium Oscillatoria Formosa, World Journal of Microbiology \& Biotechnology, 27(9). 2139-2146. September 2011.

[30] Wang, X., Shao, C.G., Liu, L., Guo, X., Xu, Y.M., Lv, X. Optimization, partial characterization and antioxidant activity of an exopolysaccharide from Lactobacillus plantarum KX041, International Journal of Biological Macromolecules, 103. 11731184. October 2017

[31] Imran, M.Y.M., Reehana, N., Jayaraj, K.A., Ahamed, A.A.P., Dhanasekaran, D., Thajuddin, N., Alharbi, N.S., Muralitharan, G., Statistical optimization of exopolysaccharide production by Lactobacillus plantarum NTMI05 and NTMI20, International Journal of Biological Macromolecules, 93. 731-745. December 2016.

[32] Cerning, J., Bouillanne, C., Landon, M., Desmazeaud, M., Isolation and Characterization of Exopolysaccharides from SlimeForming Mesophilic Lactic Acid Bacteria, Journal of Dairy Science, 75(3). 692-699. March 1992.

[33] Tao, J., Xu, S.X., Meng, D.J., Zhang, X.D., Su, Z., Zong, W., The conditions optimization of Lactobacillus exopolysaccharides from fermentation, The Food Industry, 38(1). 28-31. January 2017.

[34] Ibarburu, I., Puertas, A.I., Berregi, I., Rodriguez-Carvajal, M.A., Prieto, A., Duenas, M.T., Production and partial characterization of exopolysaccharides produced by two Lactobacillus suebicus strains isolated from cider, International Journal of Food Microbiology, 214. 54-62. December 2015.

[35] Wang, C.L., Huang, T.H., Liang, T.W., Fang, C.Y., Wang, S.L., Production and characterization of exopolysaccharides and antioxidant from Paenibacillus sp. TKU023, New Biotechnology, 28(6). 559-565. October 2011 